Document downloaded from:

http://hdl.handle.net/10251/43593

This paper must be cited as:

Martínez Jiménez, F.; Oprocha, P.; Peris Manguillot, A. (2013). Distributional chaos for operators with full scrambled sets. Mathematische Zeitschrift. 274(1-2):603-612. doi:10.1007/s00209-012-1087-8.

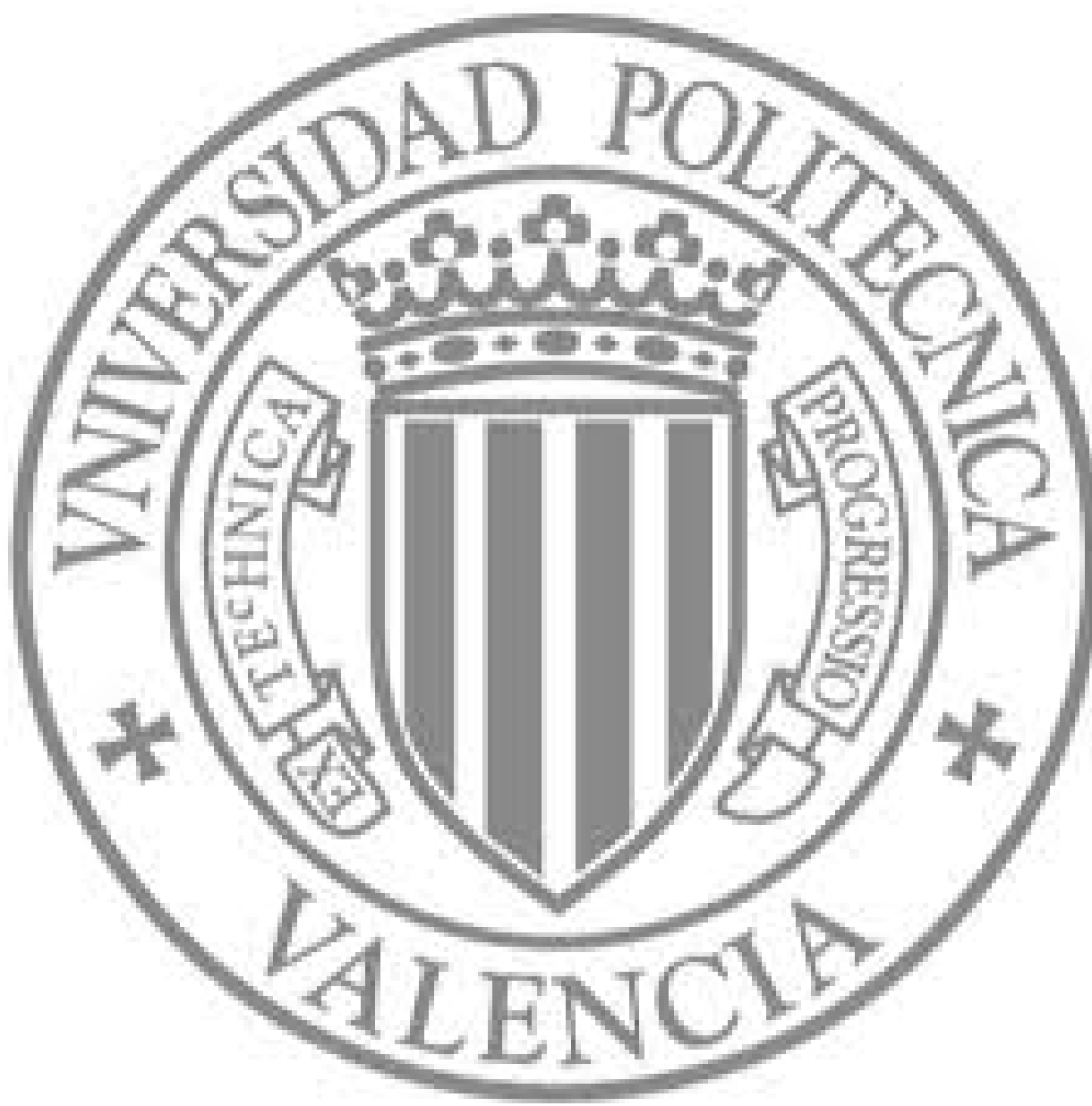

The final publication is available at

http://dx.doi.org/10.1007/s00209-012-1087-8

Copyright Springer Verlag (Germany) 


\title{
DISTRIBUTIONAL CHAOS FOR OPERATORS WITH FULL SCRAMBLED SETS
}

\author{
FÉLIX MARTÍNEZ-GIMÉNEZ, PIOTR OPROCHA, AND ALFREDO PERIS
}

\begin{abstract}
In this article we answer in the negative the question of whether hypercyclicity is sufficient for distributional chaos for a continuous linear operator (we even prove that the mixing property does not suffice). Moreover, we show that a extremal situation is possible: There are (hypercyclic and non-hypercyclic) operators such that the whole space consists, except zero, of distributionally irregular vectors.
\end{abstract}

\section{INTRODUCTION}

In recent years many researchers were looking for conditions that yield complex, nontrivial dynamics of linear operators (note that, to admit such behaviour, the space must be infinite dimensional). Probably the most studied is the notion of the hypercyclicity, that is, the existence of vectors $x \in X$ such that the orbit of this vector $x, T(x), T^{2}(x), \ldots$ under action of a continuous and linear operator $T: X \rightarrow X$ on a topological vector space (most often Banach or Fréchet space) $X$ forms a dense subset of $X$. We refer the reader to the recent books [5] and [10] for an accessible introduction into the topic together with the review on the history of this problem. After publication of the book of Devaney [7] studies on hypercyclicity became essential tool for understanding of chaos in the sense of Devaney, since definition of chaos from [7] in our context requires hypercyclicity and density of the set of periodic points of $T$ in $X$ (see [1]).

Another definition of chaos was born a few years before [7], when Li and Yorke published their famous paper on the study of complicated dynamics of maps on the unit interval [13]. In contrast to the definition of Devaney, the definition derived from [13] concentrates rather on local aspects of dynamics of pairs than a complex global behavior induced by hypercyclicity.

Distributional chaos was introduced by Schweizer and Smital in [18] as a natural extension of the notion of chaos given several years before by Li and Yorke in [13]. This concept will be the main notion considered in this paper. Following [14], we will consider only the definition of uniform distributional chaos, which is one of the strongest possibilities (see [16]).

Recall that, if $A \subset \mathbb{N}$, then its upper density is the number

$$
\overline{\operatorname{dens}}(A)=\limsup _{n \rightarrow \infty} \frac{1}{n}|\{i<n ; i \in A\}|,
$$

where $|S|$ denotes cardinality of the set $S$. Using this notation, distributional chaos can be defined as follows:

2010 Mathematics Subject Classification. 47A16, 37D45.

Key words and phrases. Distributional chaos, hypercyclic operators, irregular vectors. 
Definition 1.1. Let $f$ be a continuous self map on a metric space $(X, d)$. If there exists an uncountable set $D \subset X$ and $\varepsilon>0$ such that for every $t>0$ and every distinct $x, y \in D$ the following conditions hold:

$$
\begin{aligned}
& \overline{\operatorname{dens}}\left\{i \in \mathbb{N} ; d\left(f^{i}(x), f^{i}(y)\right) \geq \varepsilon\right\}=1, \\
& \overline{\operatorname{dens}}\left\{i \in \mathbb{N} ; d\left(f^{i}(x), f^{i}(y)\right)<t\right\}=1,
\end{aligned}
$$

then we say that $f$ exhibits uniform distributional chaos. The set $D$ is called a distributionally $\varepsilon$-scrambled set.

Let $X$ be a Banach space and let $T: X \rightarrow X$ be an operator. While there is lack of full characterization of distributional chaos for operators, it is possible to provide effective criteria ensuring this property $[4,11,14]$. It was proved in [14] that if a weighted backward shift on $\ell^{p}$-space is chaotic in the sense of Devaney then it also exhibits distributional chaos. There were also examples of distributionally chaotic operators which are not hypercyclic. So the only uncertain possibility left in [14] is the question whether hypercyclicity is sufficient for distributional chaos. The negative answer to this question is given in Section 2. Even more is shown, that is, mixing is not enough for distributional chaos. Recall that $T$ is mixing if the set $\left\{n \in \mathbb{N} ; T^{n}(U) \cap V \neq \emptyset\right\}$ is co-finite for every pair of non-empty open sets $U, V$.

We recall from [3] that a vector $x \in X$ is said to be irregular for an operator $T$ if $\liminf _{n \rightarrow \infty}\left\|T^{n} x\right\|=0$, while $\lim \sup _{n \rightarrow \infty}\left\|T^{n} x\right\|=\infty$. Inspired by this definition, Bermúdez et al. introduced in [4] the following notion:

Definition 1.2. A vector $x \in X$ is said to be distributionally irregular for $T$ if there are increasing sequences of integers $A=\left\{n_{k} ; k \in \mathbb{N}\right\}$ and $B=\left\{m_{k} ; k \in \mathbb{N}\right\}$ such that $\overline{\operatorname{dens}}(A)=\overline{\operatorname{dens}}(B)=1, \lim _{k \rightarrow \infty}\left\|T^{n_{k}} x\right\|=0$ and $\lim _{k \rightarrow \infty}\left\|T^{m_{k}} x\right\|=\infty$.

In [4] the authors proved that the existence of irregular vectors is equivalent to admitting Li-Yorke pairs. The same paper shows that every infinite dimensional separable Banach space admits a hypercyclic and distributionally chaotic operator which has a dense distributionally irregular scrambled set $D$, that is distributionally scrambled set such that each vector is distributionally irregular. Then the natural question which immediately arises is whether it is possible that $D=X$, i.e. $D$ is not only dense but equals to the whole space.

Definition 1.3. We say that an operator $T$ is completely distributionally irregular if every vector $x \in X \backslash\{0\}$ is distributionally irregular.

Remark 1.4. Note that if $T$ is completely distributionally irregular then the whole space is distributionally $\varepsilon$-scrambled, for any $\varepsilon>0$.

In Section 3 we will show that there exist completely distributionally irregular operators (even with completely distributionally irregular inverse) and that this property is independent of hypercyclicity or distributional irregularity of the inverse.

We refer the reader to $[2,12,17,19]$ for other recent works on distributional chaos for operators. Also, in [15] it is shown that there are completely distributionally irregular operators $T$ such that the sequence $\left(\left\|T^{n}\right\|\right)_{n}$ is increasing. 


\section{Mixing AND Distributional CHAOS}

In this section we will consider the unilateral backward shift $(B x)_{i}=x_{i+1}$ on the weighted $\ell^{p}$-space

$$
\ell^{p}(v):=\left\{x=\left(x_{j}\right)_{j \in \mathbb{N}} ;\|x\|^{p}=\sum_{j \in \mathbb{N}}\left|x_{j}\right|^{p} v_{j}<\infty\right\} .
$$

The map $B: \ell^{p}(v) \rightarrow \ell^{p}(v)$ is well-defined (and, equivalently, continuous) if and only if $\sup _{j} \frac{v_{j}}{v_{j+1}}<\infty$. We will show that there are sequences of weights so that unilateral shift is mixing but not distributionally chaotic. We should point out that it is easy to construct non-hypercyclic but distributionally chaotic unilateral shifts (see [14]).

Theorem 2.1. Let $n_{k}:=(k !)^{3}, k \in \mathbb{N}$, and let $v=\left(v_{j}\right)_{j}$ be the sequence of weights given by $v_{j}=k^{-1}$ for $n_{k} \leq j<n_{k+1}, k \in \mathbb{N}$. Then the operator $B$ is mixing on $X:=\ell^{p}(v), 1 \leq p<\infty$, but $T$ is not distributionally chaotic.

Proof. The fact that $B$ is mixing can be deduced from [6] (see also Chapter 4 in [10] for more details), since $\lim _{j} v_{j}=0$.

We will show that, for each $x \in X$ and for every $\varepsilon \in] 0,1[$,

$$
\lim _{n \rightarrow \infty} \frac{\left|\left\{j \leq n ;\left\|B^{j} x\right\|<\varepsilon\right\}\right|}{n}=1
$$

that excludes the possibility of existence of distributionally chaotic pairs.

We fix an integer $k_{0}>6$ satisfying

$$
\sum_{j \geq n_{k_{0}}}\left|x_{j}\right|^{p} v_{j}<\varepsilon / 4 \text { and } k_{0}^{-1}<\varepsilon / 4
$$

If $n \geq n_{k_{0}+1}$, let $k \geq k_{0}$ with $n_{k+1} \leq n<n_{k+2}$. We can write $n=N n_{k}+m$ with $m, N \in \mathbb{N}, m \leq n_{k}, N>k^{3}$. Since

$$
\sum_{i=1}^{N-1}\left(\sum_{j=i n_{k}}^{(i+1) n_{k}-1}\left|x_{j}\right|^{p} v_{j}\right) \leq \sum_{j \geq n_{k}}\left|x_{j}\right|^{p} v_{j}<\varepsilon / 4<1
$$

then, for

$$
I:=\left\{i<N ; \sum_{j=i n_{k}}^{(i+1) n_{k}-1}\left|x_{j}\right|^{p} v_{j} \geq k^{-2}\right\}
$$

we have $|I| \leq k^{2}$. Thus,

$$
|\{1, \ldots, N-1\} \backslash I| \geq N-1-k^{2} .
$$

If $i \in J:=\{1, \ldots, N-1\} \backslash I$, then

$$
\sum_{j=i n_{k}}^{(i+1) n_{k}-1}\left|x_{j}\right|^{p} \leq(k+1) \sum_{j=i n_{k}}^{(i+1) n_{k}-1}\left|x_{j}\right|^{p} v_{j}<(k+1) k^{-2}<\varepsilon / 2,
$$

by the definition of $I$, and since $v_{j}=(k+1)^{-1}$ for $n_{k+1} \leq j<n_{k+2}$. This implies that, if we fix $i \in J$ and $j \in\left[i n_{k},(i+1) n_{k}-n_{k-1}\right]$, then

$$
\left\|B^{j-1} x\right\|^{p}=\sum_{l=j}^{(i+1) n_{k}-1}\left|x_{l}\right|^{p} v_{l-j+1}+\sum_{l \geq(i+1) n_{k}}\left|x_{l}\right|^{p} v_{l-j+1}
$$




$$
\leq \sum_{l=i n_{k}}^{(i+1) n_{k}-1}\left|x_{l}\right|^{p}+\sum_{l \geq(i+1) n_{k}}\left|x_{l}\right|^{p} v_{l} \frac{v_{l-j+1}}{v_{l}}<\frac{\varepsilon}{2}+\frac{3 \varepsilon}{8}<\varepsilon,
$$

since $j<n_{k+2}$ and $l-j+1 \geq n_{k-1}$ whenever $l \geq(i+1) n_{k}$, and by taking into account that $v_{s} / v_{r} \leq(k+2) /(k-1) \leq 3 / 2$ if $r>s \geq n_{k-1}$ and $r-s<n_{k+2}$. Therefore,

$$
\begin{gathered}
\frac{\left|\left\{j \leq n ;\left\|B^{j} x\right\|^{p}<\varepsilon\right\}\right|}{n} \geq \frac{\sum_{i \in J}\left(n_{k}-n_{k-1}\right)}{n} \geq \frac{\left(N-1-k^{2}\right)\left(n_{k}-n_{k-1}\right)}{n} \\
\geq\left(\frac{N-1-k^{2}}{N+1}\right)\left(\frac{n_{k}-n_{k-1}}{n_{k}}\right)>\left(1-\frac{k^{2}+2}{k^{3}+1}\right)\left(1-\frac{1}{k^{3}}\right) \underset{k \rightarrow \infty}{\longrightarrow} 1 .
\end{gathered}
$$

Remark 2.2. Observe that we have shown in Theorem 2.1 that

$$
\overline{\operatorname{dens}}\left\{j: d\left(B^{j} x, B^{j} y\right) \geq \varepsilon\right\}=0
$$

for every $x, y \in X, x \neq y$, and for every $\varepsilon>0$. In other words, the mixing rate of $B$ is very slow.

\section{FULL DISTRIBUTIONALLY SCRAMBLED SETS AND HYPERCYCLICITY}

In this section we will consider the bilateral forward shift $(T x)_{i}=x_{i-1}$ and the backward shift $(B x)_{i}=x_{i+1}$ on the weighted $\ell^{p}$-space

$$
\ell^{p}(v, \mathbb{Z}):=\left\{x=\left(x_{j}\right)_{j \in \mathbb{Z}} ;\|x\|^{p}=\sum_{j \in \mathbb{Z}}\left|x_{j}\right|^{p} v_{j}<\infty\right\},
$$

where the weight sequence $v=\left(v_{j}\right)_{j \in \mathbb{Z}}$ will be constructed satisfying certain general assumptions. We also recall that $B: \ell^{p}(v, \mathbb{Z}) \rightarrow \ell^{p}(v, \mathbb{Z})$ (respectively, $T:$ $\left.\ell^{p}(v, \mathbb{Z}) \rightarrow \ell^{p}(v, \mathbb{Z})\right)$ is well-defined (equivalently, continuous) if and only if $\sup _{j \in \mathbb{Z}} \frac{v_{j}}{v_{j+1}}<$ $\infty$ (respectively, $\sup _{j \in \mathbb{Z}} \frac{v_{j}}{v_{j-1}}<\infty$ ). Our purpose is to provide examples of weight sequences $v$ such that every non-zero vector is distributionally irregular for $T: \ell^{p}(v, \mathbb{Z}) \rightarrow$ $\ell^{p}(v, \mathbb{Z})$, and for some of these examples $T^{-1}=B$ will have the same property. We also study its relation to hypercyclicity. In what follows, we will simply write $a_{j}^{i}=\left(a_{j}\right)^{i}$. We hope it will cause no confusion to the reader.

Theorem 3.1. Let $v=\left(v_{j}\right)_{j \in \mathbb{Z}}$ be a weight sequence that satisfies the following conditions:

(1) there are sequences of integers $\left(n_{j}\right)_{j \in \mathbb{Z}}$ and $\left(m_{j}\right)_{j \in \mathbb{Z}}$ with $n_{j}<m_{j}<n_{j+1}$, $j \in \mathbb{Z}$, and $M>1$ such that $M v_{m_{-k}} \geq v_{j}$ for every $j \in\left[m_{-k}, m_{k-1}\right], k \in \mathbb{N}$, and if we consider

$$
\left.\left.S_{k}:=\sup \left\{\frac{v_{j}}{v_{j-1}} ; j \notin\right] m_{-k}, m_{k-1}\right]\right\}, \quad k \in \mathbb{N},
$$

then for every $\varepsilon>0$ we find $k \in \mathbb{N}$ with $v_{n_{k}}<\varepsilon$ and

$$
S_{k}^{k\left(n_{k}-m_{-k}\right)} \leq \min \left\{M, \frac{\min \left\{v_{i} ; m_{-k} \leq i \leq m_{k-1}\right\}}{v_{n_{k}}}\right\},
$$

(2) for every $N \in \mathbb{N}$, there exists $k \in \mathbb{N}$ such that $v_{j}>N$, for $k \leq j \leq N k$.

Then the forward shift $T: \ell^{p}(v, \mathbb{Z}) \rightarrow \ell^{p}(v, \mathbb{Z})$ is completely distributionally irregular. 
Proof. Let $x \in \ell^{p}(v, \mathbb{Z})$ be an arbitrary non-zero vector. Given $M>1$ satisfying condition (1), and an arbitrary $\delta>0$, we fix $m \in \mathbb{N}$ such that

$$
\sum_{|j|>m}\left|x_{j}\right|^{p} v_{j}<\frac{\delta}{2\left(1+M^{2}\right)}
$$

Again by condition (1) there exists $k \in \mathbb{N}$ with $v_{n_{k}}<\varepsilon:=\delta \frac{\min \left\{v_{j} ;|j| \leq m\right\}}{2 M\left(1+\|x\|^{p}\right)}$, and such that $[-m, m] \subset] m_{-k}, m_{k-1}\left[\right.$. For any $l \in\left[n_{k}-m_{-k}, k\left(n_{k}-m_{-k}\right)\right]$ we have

$$
\left\|T^{l} x\right\|^{p}=\sum_{j \in\left[m_{-k}, m_{k-1}\right]}\left|x_{j}\right|^{p} v_{j+l}+\sum_{j \notin\left[m_{-k}, m_{k-1}\right]}\left|x_{j}\right|^{p} v_{j+l} .
$$

For the first summand we obtain

$$
\begin{gathered}
\sum_{j \in\left[m_{-k}, m_{k-1}\right]}\left|x_{j}\right|^{p} v_{j+l} \leq \sum_{|j| \leq m}\left|x_{j}\right|^{p} v_{j} \frac{v_{j+l}}{v_{j}}+\sum_{j \in\left[m_{-k}, m_{k-1}\right] \backslash[-m, m]}\left|x_{j}\right|^{p} v_{j} \frac{v_{j+l}}{v_{j}} \\
\leq \sum_{|j| \leq m}\left|x_{j}\right|^{p} v_{j} \frac{v_{n_{k}}\left(\prod_{i=n_{k}}^{j+l-1} \frac{v_{i+1}}{v_{i}}\right)}{v_{j}}+\sum_{j \in\left[m_{-k}, m_{k-1}\right] \backslash[-m, m]}\left|x_{j}\right|^{p} v_{j} \frac{v_{j+l}}{v_{j}} \\
\leq\|x\|^{p} \frac{v_{n_{k}} S_{k}^{m+l-n_{k}}}{\min \left\{v_{j} ;|j| \leq m\right\}}+\frac{\delta}{2\left(1+M^{2}\right)} \max \left\{\frac{v_{j+l}}{v_{j}} ; j \in\left[m_{-k}, m_{k-1}\right] \backslash[-m, m]\right\} \\
<\|x\|^{p} \frac{\delta M}{2 M\left(1+\|x\|^{p}\right)}+\frac{\delta}{2\left(1+M^{2}\right)} \frac{v_{n_{k}} S_{k}^{k\left(n_{k}-m_{-k}\right)}}{\min \left\{v_{j} ; m_{-k} \leq j \leq m_{k-1}\right\}} \leq \frac{\delta\left(2+M^{2}\right)}{2\left(1+M^{2}\right)} .
\end{gathered}
$$

by the selection of $m$ and $\varepsilon$. With respect to the second summand in (3.1), we get

$$
\begin{aligned}
& \sum_{j \notin\left[m_{-k}, m_{k-1}\right]}\left|x_{j}\right|^{p} v_{j+l}=\sum_{m_{-k}-l \leq j<m_{-k}}\left|x_{j}\right|^{p} v_{j} \frac{v_{j+l}}{v_{j}}+\sum_{j \notin\left[m_{-k}-l, m_{k-1}\right]}\left|x_{j}\right|^{p} v_{j} \frac{v_{j+l}}{v_{j}} \\
\leq & \left(\sum_{m_{-k}-l \leq j<m_{-k}}\left|x_{j}\right|^{p} v_{j}\right) M S_{k}^{l}+\left(\sum_{j \notin\left[m_{-k}-l, m_{k-1}\right]}\left|x_{j}\right|^{p} v_{j}\right) S_{k}^{l}<\frac{\delta M^{2}}{2\left(1+M^{2}\right)} .
\end{aligned}
$$

Therefore we have $\left\|T^{l} x\right\|^{p}<\delta$, and we obtain the existence of $A=\left\{l_{j} ; j \in \mathbb{N}\right\} \subset \mathbb{N}$ such that $\overline{\operatorname{dens}}(A)=1$ and $\lim _{j}\left\|T^{l_{j}} x\right\|=0$.

On the other hand, since $x \neq 0$, there is $i_{0} \in \mathbb{Z}$ such that $x_{i_{0}} \neq 0$. By condition (2), given any $N \in \mathbb{N}$ there is $k \in \mathbb{N}$ such that $v_{j}>N$ for all $j \in[k, N k]$. Without loss of generality we may assume $N>\max _{j \leq\left|i_{0}\right|} v_{j}$, so that $k>i_{0}$, therefore $\left\|T^{r} x\right\| \geq\left|x_{i_{0}}\right| N^{1 / p}$ for each $r \in\left[k-i_{0}, N k-i_{0}\right]$, which yields that there is $B=$ $\left\{r_{j} ; j \in \mathbb{N}\right\} \subset \mathbb{N}$ such that $\overline{\operatorname{dens}}(B)=1$ and $\lim _{j}\left\|T^{r_{j}} x\right\|=\infty$.

As a consequence of Theorem 3.1 we obtain the analogous result for the backward shift.

Corollary 3.2. Let $v=\left(v_{j}\right)_{j \in \mathbb{Z}}$ be a weight sequence that satisfies the following conditions:

(1) there are sequences of integers $\left(n_{j}\right)_{j \in \mathbb{Z}}$ and $\left(m_{j}\right)_{j \in \mathbb{Z}}$ with $n_{j}<m_{j}<n_{j+1}$, $j \in \mathbb{Z}$, and $M>1$ such that $M v_{m_{k-1}} \geq v_{j}$ for every $j \in\left[m_{-k}, m_{k-1}\right]$, $k \in \mathbb{N}$, and if we consider

$$
\left.\left.s_{k}:=\inf \left\{\frac{v_{j}}{v_{j-1}} ; j \notin\right] m_{-k}, m_{k-1}\right]\right\}, k \in \mathbb{N},
$$


then for every $\varepsilon>0$ we find $k \in \mathbb{N}$ with $v_{n_{-k}}<\varepsilon$ and

$$
s_{k}^{k\left(n_{-k}-m_{k-1}\right)} \leq \min \left\{M, \frac{\min \left\{v_{i} ; m_{-k} \leq i \leq m_{k-1}\right\}}{v_{n_{-k}}}\right\},
$$

(2) for every $N \in \mathbb{N}$, there exits $k \in \mathbb{N}$ such that $v_{j}>N$, for $-N k \leq j \leq-k$. Then the backward shift $B=T^{-1}: \ell^{p}(v, \mathbb{Z}) \rightarrow \ell^{p}(v, \mathbb{Z})$ is completely distributionally irregular.

Proof. It suffices to consider the isomorphism $\left(x_{j}\right)_{j \in \mathbb{Z}} \mapsto\left(x_{-j}\right)_{j \in \mathbb{Z}}$ that conjugates $B$ to the forward shift $T: \ell^{p}\left(v^{\prime}, \mathbb{Z}\right) \rightarrow \ell^{p}\left(v^{\prime}, \mathbb{Z}\right)$, where the weight sequence $v^{\prime}$ is defined as $v_{j}^{\prime}=v_{-j}$ and the necessary sequences to apply Theorem 3.1 are defined by $n_{j}^{\prime}:=-n_{-j}, m_{j}^{\prime}:=-m_{-j-1}$.

For convenience of the constructions that we will provide in the final examples, we need a further result which is a consequence of Corollary 3.2 by simply applying a shift $m_{k}^{\prime}=m_{k+1}, n_{k}^{\prime}=n_{k+1}$, on the right part of the sequences $\left(m_{k}\right)_{k}$ and $\left(n_{k}\right)_{k}$ $(k \geq 0)$, and leaving invariant the left parts $m_{k}^{\prime}=m_{k}, n_{k}^{\prime}=n_{k}, k<0$.

Corollary 3.3. Let $v=\left(v_{j}\right)_{j \in \mathbb{Z}}$ be a weight sequence that satisfies the following conditions:

(1) there are sequences of integers $\left(n_{j}\right)_{j \in \mathbb{Z}}$ and $\left(m_{j}\right)_{j \in \mathbb{Z}}$ with $n_{j}<m_{j}<n_{j+1}$, $j \in \mathbb{Z}$, and $M>1$ such that $M v_{m_{k}} \geq v_{j}$ for every $j \in\left[m_{-k}, m_{k}\right], k \in \mathbb{N}$, and if we consider

$$
\left.\left.s_{k}:=\inf \left\{\frac{v_{j}}{v_{j-1}} ; j \notin\right] m_{-k}, m_{k}\right]\right\}, k \in \mathbb{N},
$$

then for every $\varepsilon>0$ we find $k \in \mathbb{N}$ with $v_{n_{-k}}<\varepsilon$ and

$$
s_{k}^{k\left(n_{-k}-m_{k}\right)} \leq \min \left\{M, \frac{\min \left\{v_{i} ; m_{-k} \leq i \leq m_{k}\right\}}{v_{n_{-k}}}\right\},
$$

(2) for every $N \in \mathbb{N}$, there exits $k \in \mathbb{N}$ such that $v_{j}>N$, for $-N k \leq j \leq-k$. Then the backward shift $B=T^{-1}: \ell^{p}(v, \mathbb{Z}) \rightarrow \ell^{p}(v, \mathbb{Z})$ is completely distributionally irregular.

With the above results, we are ready to provide the desired examples. In all of them the sequences of integers $\left(n_{j}\right)_{j \in \mathbb{Z}}$ and $\left(m_{j}\right)_{j \in \mathbb{Z}}$ with $n_{j}<m_{j}<n_{j+1}, j \in \mathbb{Z}$ are such that for every $k \in \mathbb{Z}$ we have:

$$
v_{j-1} \leq v_{j} \text { when } n_{k}<j \leq m_{k} \text {, and } v_{j-1} \geq v_{j} \text { when } m_{k}<j \leq n_{k+1} \text {. }
$$

Generally speaking, the positions $v_{m_{k}}$ represent "hills" of the weight sequence, and the positions $v_{n_{k}}$ are "valleys".

Example 3.4. We will select $v$ such that $T$ and $T^{-1}$ are completely distributionally irregular on $\ell^{p}(v, \mathbb{Z})$, but $T$ is not hypercyclic. First, we put some general conditions which will lead to inductive construction of sequences of integers $\left(m_{k}\right)_{k \in \mathbb{Z}}$ and $\left(n_{k}\right)_{k \in \mathbb{Z}}$ with the desired properties. We will require that sequences $\left(m_{k}\right)_{k \in \mathbb{Z}}$, $\left(n_{k}\right)_{k \in \mathbb{Z}}$ increase fast enough so that they satisfy the following conditions:

(a) $m_{0}=1, n_{1}=4, v_{m_{0}}=2, v_{n_{1}}=2^{-2}$,

(b) $v_{n_{k}}=2^{-2 k}, v_{m_{k}}=2^{2 k+1}, k \in \mathbb{N}, v_{i} / v_{i-1}=v_{j} / v_{j-1}$ if $\left.\left.i, j \in\right] n_{k}, m_{k}\right]$, or if $\left.i, j \in] m_{k-1}, n_{k}\right], k \in \mathbb{N}$,

(c) $m_{k}-n_{k}>2\left(m_{k-1}-n_{k-1}\right), n_{k+1}-m_{k}>2\left(n_{k}-m_{k-1}\right), k \in \mathbb{N}$, and

(d) $v_{-j}=v_{j}^{-1}, j \in \mathbb{N}, m_{k}=-n_{-k}, k \in \mathbb{Z}$. 


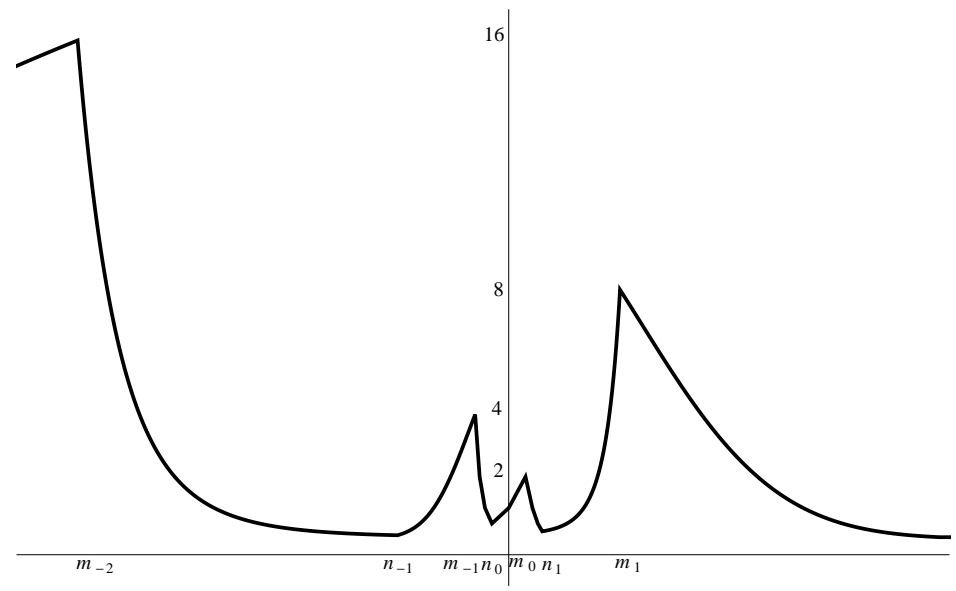

FIGURE 1. Example 3.4

Observe that conditions (b) and (d) give that $\frac{\min \left\{v_{i} ; m_{-k} \leq i \leq m_{k-1}\right\}}{v_{n_{k}}}=$ $\frac{v_{n_{-k+1}}}{v_{n_{k}}}=2$ for every $k \in \mathbb{N}$, and the supremum of the slope of $v$ outside the interval $\left[m_{-k}, m_{k-1}\right]$ is $S_{k}=v_{j} / v_{j-1}$ for any $n_{k}<j \leq m_{k}, k \in \mathbb{N}$, by the all the assumptions. In order to fulfill condition (1) in Theorem 3.1, we set $M=2$ and $S_{k}=2^{1 / k\left(n_{k}-m_{-k}\right)}=2^{1 /\left(2 k n_{k}\right)}, k \in \mathbb{N}$. Thus, we get $S_{k}^{m_{k}-n_{k}}=v_{m_{k}} / v_{n_{k}}=2^{4 k+1}$, which implies $m_{k}=\left(8 k^{2}+2 k+1\right) n_{k}, k \in \mathbb{N}$.

Analogously, $\frac{\min \left\{v_{i} ; m_{-k} \leq i \leq m_{k}\right\}}{v_{n_{-k}}}=\frac{v_{n_{k}}}{v_{n_{-k}}}=2$ for every $k \in \mathbb{N}$, and the infimum of the slope of $v$ outside the interval $\left[m_{-k}, m_{k}\right]$ is $s_{k}=v_{j} / v_{j-1}$ for any $m_{k}<j \leq n_{k+1}, k \in \mathbb{N}$. Again, to have condition (1) in Corollary 3.3, we set $M=2$ and $s_{k}=2^{1 / k\left(n_{-k}-m_{k}\right)}=2^{-1 /\left(2 k m_{k}\right)}, k \in \mathbb{N}$. As a consequence, $s_{k}^{n_{k+1}-m_{k}}=$ $v_{n_{k+1}} / v_{m_{k}}=2^{-4 k-3}$, which yields $n_{k+1}=\left(8 k^{2}+6 k+1\right) m_{k}, k \in \mathbb{N}$. This allows us

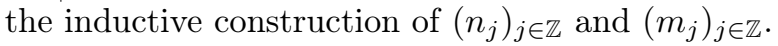

To check condition (2) in Theorem 3.1 and Corollary 3.3, we notice that

$$
v_{r}=2^{2 k+1} s_{k}^{r-m_{k}}=2^{2 k+1} 2^{-\frac{r-m_{k}}{2 k m_{k}}}>2^{2 k} \text { if } m_{k} \leq r \leq 2 k m_{k},
$$

and that

$$
v_{r}=2^{2 k} S_{k}^{m_{-k}-r}=2^{2 k} 2^{\frac{-n_{k}-r}{2 k n_{k}}}>2^{2 k-1} \text { if } 2 k m_{-k} \leq r \leq m_{-k} .
$$

This implies that all the conditions in Theorem 3.1 and Corollary 3.3 are satisfied and so $T$ and $T^{-1}$ are completely distributionally irregular.

Finally, since $v_{j}=v_{-j}^{-1}$ for all $j \in \mathbb{Z}$, there is no increasing sequence $\left(j_{k}\right)_{k}$ in $\mathbb{N}$ such that $\lim _{k} v_{j_{k}}=\lim _{k} v_{-j_{k}}=0$, which avoids the hypercyclicity of $T$ (See, e.g., Theorem 2 in [9]).

Example 3.5. Our purpose now is to construct $v$ such that $T$ and $T^{-1}$ are completely distributionally irregular on $\ell^{p}(v, \mathbb{Z})$, and $T$ is hypercyclic. To do so, we set up the following preliminary conditions on the weights

(a) $n_{1}=4, m_{-1}=-4, v_{n_{0}}=1, v_{m_{0}}=2, v_{n_{1}}=2^{-1}, v_{m_{-1}}=2$, and 
(b) $m_{k}=-n_{-k}, k \in \mathbb{Z}, v_{n_{k}}=2^{-2 k+1}, v_{m_{k}}=2^{k+1}, v_{n_{-k}}=2^{-2 k}, v_{m_{-k}}=2^{k}$, $k \in \mathbb{N}, v_{i} / v_{i-1}=v_{j} / v_{j-1}$ if $\left.\left.i, j \in\right] n_{k}, m_{k}\right]$, or if $\left.\left.i, j \in\right] m_{k-1}, n_{k}\right], k \in \mathbb{Z}$, and

(c) $m_{k}-n_{k}>2\left(m_{k-1}-n_{k-1}\right), n_{k+1}-m_{k}>2\left(n_{k}-m_{k-1}\right), k \in \mathbb{N}$.

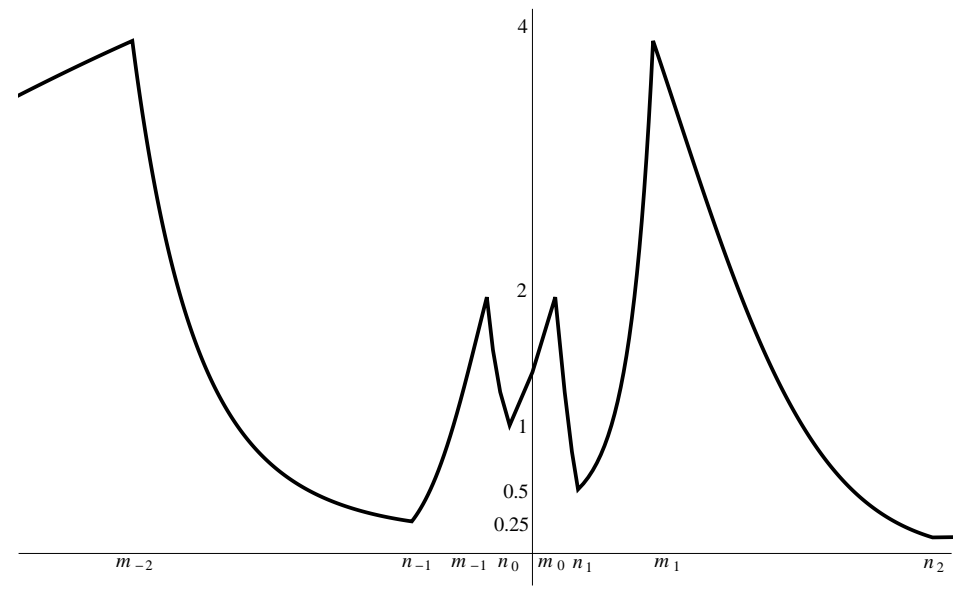

FiguRE 2. Example 3.5

We will check again that the hypothesis of Theorem 3.1 and Corollary 3.3 are satisfied. Condition (b) gives $\frac{\min \left\{v_{i} ; m_{-k} \leq i \leq m_{k-1}\right\}}{v_{n_{k}}}=\frac{v_{n_{-k+1}}}{v_{n_{k}}}=2$ for every $k \in \mathbb{N}$, and the supremum of the slope of $v$ outside the interval $\left[m_{-k}, m_{k-1}\right]$ is $S_{k}=v_{j} / v_{j-1}$ for any $n_{k}<j \leq m_{k}, k \in \mathbb{N}$. As in the previous example, we set $M=2$ and $S_{k}=2^{1 / k\left(n_{k}-m_{-k}\right)}=2^{1 /\left(2 k n_{k}\right)}, k \in \mathbb{N}$. Thus, $S_{k}^{m_{k}-n_{k}}=v_{m_{k}} / v_{n_{k}}=2^{3 k}$, which implies $m_{k}=\left(6 k^{2}+1\right) n_{k}, k \in \mathbb{N}$.

Analogously, $\frac{\min \left\{v_{i} ; m_{-k} \leq i \leq m_{k}\right\}}{v_{n_{-k}}}=\frac{v_{n_{k}}}{v_{n_{-k}}}=2$ for every $k \in \mathbb{N}$, and the infimum of the slope of $v$ outside the interval $\left[m_{-k}, m_{k}\right]$ is $s_{k}=v_{j} / v_{j-1}$ for any $m_{k}<j \leq n_{k+1}, k \in \mathbb{N}$. Again, we set $M=2$ and $s_{k}=2^{1 / k\left(n_{-k}-m_{k}\right)}=2^{-1 /\left(2 k m_{k}\right)}$, $k \in \mathbb{N}$. In consequence, $s_{k}^{n_{k+1}-m_{k}}=v_{n_{k+1}} / v_{m_{k}}=2^{-3 k-2}$, that yields $n_{k+1}=$ $\left(6 k^{2}+4 k+1\right) m_{k}, k \in \mathbb{N}$.

To check the final conditions for $T$ and $T^{-1}$ being completely distributionally irregular, we notice that

$$
v_{r}=2^{k+1} s_{k}^{r-m_{k}}=2^{k+1} 2^{-\frac{r-m_{k}}{2 k m_{k}}}>2^{k} \text { if } m_{k} \leq r \leq 2 k m_{k},
$$

and that

$$
v_{r}=2^{k} S_{k}^{m_{-k}-r}=2^{k} 2^{\frac{-n_{k}-r}{2 k n_{k}}}>2^{k-1} \text { if } 2 k m_{-k} \leq r \leq m_{-k} .
$$

For the hypercyclicity of $T$, since it is invertible, it suffices to show that there is an increasing sequence $\left(j_{k}\right)_{k}$ in $\mathbb{N}$ such that $\lim _{k} v_{j_{k}}=\lim _{k} v_{-j_{k}}=0$ (See Theorem 3.2 in [8]). Let $j_{k}:=\left(m_{k}+n_{k}\right) / 2=\left(3 k^{2}+1\right) n_{k}$. We have

$$
v_{j_{k}}=S_{k}^{j_{k}-n_{k}} v_{n_{k}}=2^{-k / 2+1},
$$


for any $k \in \mathbb{N}$. Note that $R_{k}=v_{j} / v_{j-1}$ has the same value for any $\left.\left.j \in\right] n_{-k}, m_{-k}\right]$, and thus for all $k \in \mathbb{N}$ we have $R_{k}<S_{k}$ and

$$
v_{-j_{k}}=R_{k}^{-j_{k}-n_{-k}} v_{n_{-k}}<S_{k}^{m_{k}-j_{k}} 2^{-2 k}=2^{-k / 2+1 / 2} .
$$

Indeed, $\lim _{k} v_{j_{k}}=\lim _{k} v_{-j_{k}}=0$ which concludes the hypercyclicity of $T$.

Example 3.6. We will provide $v$ such that $T$ is hypercyclic and completely distributionally irregular on $\ell^{p}(v, \mathbb{Z})$, but $T^{-1}$ is not completely distributionally irregular. We define

(a) $n_{0}=0, m_{0}=1, n_{1}=3, m_{-1}=-1, v_{n_{0}}=1, v_{m_{0}}=2, v_{n_{1}}=2^{-1}$, $v_{m_{-1}}=2$, and

(b) $m_{-k}=-m_{k-1}, n_{-k}=-m_{k}+1, n_{k+1}=m_{k}+2 k^{2}+k+1, v_{n_{-k}}=v_{n_{k}}=2^{-k}$, $v_{m_{k}}=2^{2 k^{2}}, v_{m_{-k}}=2^{-k+2}, k \in \mathbb{N}, v_{i} / v_{i-1}=v_{j} / v_{j-1}$ if $\left.\left.i, j \in\right] n_{k}, m_{k}\right]$, or if $\left.i, j \in] m_{k-1}, n_{k}\right], k \in \mathbb{Z}$, and

(c) $m_{k}-n_{k}>2\left(m_{k-1}-n_{k-1}\right), n_{k+1}-m_{k}>2\left(n_{k}-m_{k-1}\right), k \in \mathbb{N}$.

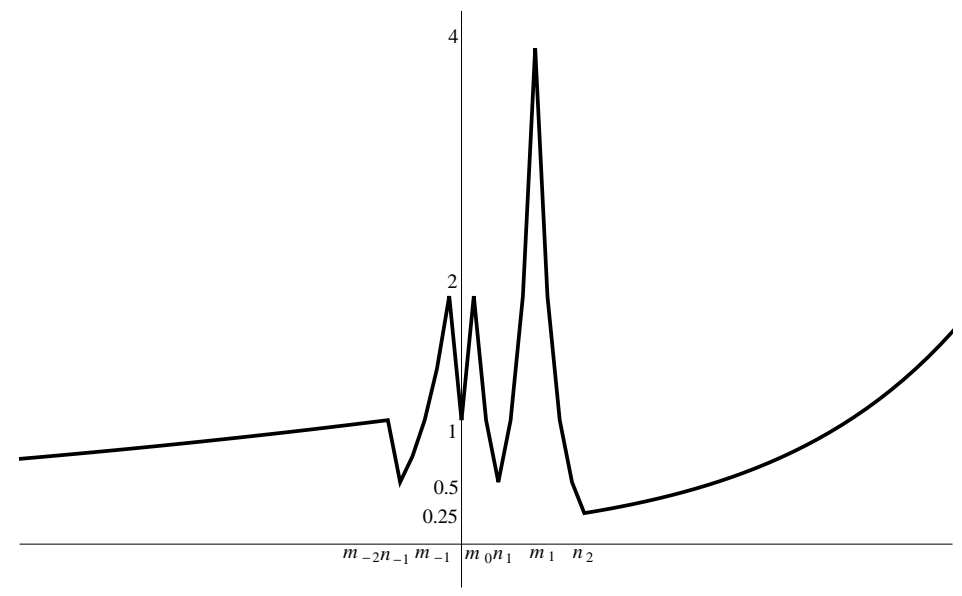

Figure 3. Example 3.6

Condition (b) gives $\frac{\min \left\{v_{i} ; m_{-k} \leq i \leq m_{k-1}\right\}}{v_{n_{k}}}=\frac{v_{n_{k-1}}}{v_{n_{k}}}=2$. We set again $M=2$ and $S_{k}=2^{1 / k\left(n_{k}-m_{-k}\right)}=2^{1 / k\left(n_{k}+m_{k-1}\right)}, k \in \mathbb{N}$. Thus, $S_{k}^{m_{k}-n_{k}}=2^{2 k^{2}+k}$, which implies $m_{k}=n_{k}+\left(2 k^{3}+k\right)\left(n_{k}+m_{k-1}\right), k \in \mathbb{N}$. We then have the formulas to construct $\left(m_{k}\right)_{k \in \mathbb{Z}},\left(n_{k}\right)_{k \in \mathbb{Z}}$, the values of the weight sequence $v$, and condition (1) of Theorem 3.1. For condition (2), pick $r_{k}:=n_{k}+2 k^{2}\left(n_{k}+m_{k-1}\right), k \in \mathbb{N}$. If $j \in\left[r_{k}, m_{k}\right]$, then $v_{j}=v_{n_{k}} S_{k}^{j-n_{k}} \geq 2^{k}$. Since $\left(m_{k}-r_{k}\right) / r_{k}>(k-1) / 2$, for each $k \in \mathbb{N}$, we obtain that $T$ is completely distributionally irregular. Moreover, $\lim _{j \rightarrow-\infty} v_{j}=0$, thus $\lim _{k} v_{n_{k}}=\lim _{k} v_{-n_{k}}=0$, and we get the hypercyclicity of $T$. Finally, if we consider $x=\left(x_{j}\right)_{j \in \mathbb{Z}}$ such that $x_{j}=1$ for $j=n_{k}, k \in \mathbb{N}$, and $x_{j}=0$ otherwise, we have $x \in \ell^{p}(v, \mathbb{Z})$ and since $n_{k}-m_{k-1} \geq k$ we also get that

$$
\left\|T^{-k} x\right\| \geq 2^{k} v_{n_{k}} \geq 1
$$

for all $k \in \mathbb{N}$. This shows that $x$ is not a distributionally irregular vector for $T^{-1}$. 


\section{ACKNOWLEDGEMENTS}

The research of first and third author was supported by MEC and FEDER, project MTM2010-14909 and by GV, Project PROMETEO/2008/101. The research of second author was supported by the Polish Ministry of Science and Higher Education from sources for science in the years 2010-2011, grant no. IP2010 029570. His research was also supported by the Marie Curie European Reintegration Grant of the European Commission under grant agreement no. PERG08-GA-2010-272297. The financial support of these institutions is hereby gratefully acknowledged. We also want to thank X. Barrachina for pointing out to us a gap in the proof of a previous version of Theorem 3.1.

\section{REFERENCES}

[1] J. Banks, J. Brooks, G. Cairns, G. Davis, and P. Stacey, On Devaney's definition of chaos, Amer. Math. Monthly 99 (1992), no. 4, 332-334.

[2] X. Barrachina and A. Peris, Distributionally chaotic translation semigroups, J. Difference Equ. Appl. 18 (2012), 751-761.

[3] B. Beauzamy, Introduction to Operator Theory and Invariant Subspaces, North-Holland, Amsterdam, 1988.

[4] T. Bermúdez, A. Bonilla, F. Martínez-Giménez, A. Peris, Li-Yorke and distributionally chaotic operators, J. Math. Anal. Appl. 373 (2011), 83-93.

[5] F. Bayart and E. Matheron, Dynamics of linear operators, Cambridge University Press, Vol. $179,2009$.

[6] G. Costakis and M. Sambarino, Topologically mixing hypercyclic operators, Proc. Amer. Math. Soc. 132 (2004), 385-389.

[7] R. L. Devaney, An introduction to chaotic dynamical systems, second ed., Addison-Wesley Studies in Nonlinearity, Addison-Wesley Publishing Company Advanced Book Program, Redwood City, CA, 1989.

[8] N. Feldman, Hypercyclicity and supercyclicity for invertible bilateral weighted shifts, Proc. Amer. Math. Soc. 131 (2003), 479-485.

[9] K.-G. Grosse-Erdmann, Hypercyclic and chaotic weighted shifts, Studia Math. 139 (2000), no. 1, 47-68.

[10] K.-G. Grosse-Erdmann and A. Peris Manguillot, Linear Chaos, Universitext, Springer, London, 2011.

[11] B. Hou, P. Cui and Y. Cao, Chaos for Cowen-Douglas operators, Proc. Amer. Math. Soc, 138 (2010), 929-936.

[12] B. Hou, G. Tian, and L. Shi, Some dynamical properties for linear operators, Illinois J. Math. 53 (2009), 857-864.

[13] T. Y. Li and J. A. Yorke, Period three implies chaos, Amer. Math. Monthly 82 (1975), no. 10, 985-992.

[14] F. Martínez-Giménez, P. Oprocha and A. Peris, Distributional chaos for backward shifts, J. Math. Anal. Appl., 351 (2009), 607-615.

[15] V. Müller and A. Peris, A problem of Beauzamy on irregular operators. Preprint 2011.

[16] P. Oprocha, Distributional chaos revisited, Trans. Amer. Math. Soc. 361 (2009), 4901-4925.

[17] P. Oprocha, A quantum harmonic oscillator and strong chaos, J. Phys. A 39 (2006), no. 47, 14559-14565.

[18] B. Schweizer and J. Smítal, Measures of chaos and a spectral decomposition of dynamical systems on the interval, Trans. Amer. Math. Soc. 344 (1994), no. 2, 737-754.

[19] X. Wu and P. Zhu, The principal measure of a quantum harmonic oscillator, J. Phys. A 44, 505101 (2011), 6 pp.

(F. Martínez-Giménez) IUMPA, Universitat Politècnica de València, Edifici 7A, Departament de Matemàtica Aplicada, E-46022 València, Spain

E-mail address: fmartinez@mat.upv.es 
(P. Oprocha) AGH University of Science and Technology, Faculty of Applied Mathematics, al. A. Mickiewicza 30, 30-059 Kraków, Poland, - and -, Institute of Mathematics, Polish Academy of Sciences, ul. Śniadeckich 8, 00-956 Warszawa, Poland

E-mail address: oprocha@agh.edu.pl

(A. Peris) IUmpa, Universitat Politècnica de València, Edifici 7A, Departament de Matemàtica Aplicada, E-46022 València, Spain

E-mail address: aperis@mat.upv.es 